\title{
Ein bisher unbekanntes Zeitzeugnis.
}

\section{Otto Warburgs Tagebuchnotizen von Februar-April 1945}

\author{
Kärin Nickelsen
}

Warburg's diary notes February-April 1945

Personal notes in the form of a diary, written by the German cell biologist Otto Warburg in the weeks before the collapse of Germany's Third Reich (Feb.-April 1945), were found at the end of one of his laboratory notebooks, which are preserved in the Archives of the Berlin-Brandenburg Academy of Sciences and Humanities. These notes are unique in form and content: there seem to be no other surviving diary notes of this type. Written from a very personal perspective, they provide a detailed account of how Warburg experienced local events in this decisive and turbulent period. Furthermore, they clearly demonstrate Warburg's embittered attitude towards some of his long-standing collaborators, whom he suspected of having denounced him to the Nazi authorities. In this paper, Warburg's notes are fully transcribed and integrated into the historical context.

Keywords: Otto Warburg, Kaiser-Wilhelm-Society, National Socialism, diary notes

Schlüsse/wörter: Otto Warburg, Kaiser-Wilhelm-Gesellschaft, Nationalsozialismus, Tagebuchnotizen

Otto Warburg (1883-1970) ist in der Wissenschaftsgeschichte vor allem bekannt für seine bahnbrechenden Arbeiten zur Zellatmung, für die ihm 1931 der Nobelpreis verliehen wurde. ${ }^{1}$ In anderen Kontexten wiederum ist er bekannt als einer der ganz wenigen Wissenschaftler jüdischer Abstammung, die während der gesamten Zeit des Nationalsozialismus in Amt und Würden blieben: Nicht nur wurde Warburg nicht deportiert, er blieb bis 1945 Direktor des Kaiser-Wilhelm-Instituts (KWI) für Zellphysiologie in Berlin-Dahlem. ${ }^{2}$ Unter anderem stand er eigenen Angaben zufolge bis 1937 unter dem persönlichen Schutz von Friedrich Glum, damals Generaldirektor der Kaiser-Wilhelm-Gesellschaft (Werner 1991: 284). Darüber hinaus hatte Warburg weitere einflussreiche Freunde in Politik und Wirtschaft, die ihn stützten so gut es ging. 1942 wurde Warburg sogar erlaubt, ein Gesuch als „jüdischer Mischling ersten Grades auf Gleichstellung mit Deutschblütigen“ zu stellen (Werner 1991: 310).

Trotzdem blieb auch Warburg nicht unbehelligt: Es gab mehrfach Situationen, die seine Stellung erheblich gefährdeten. Zu einer ernsthaften Bedrohung kam es etwa im April 1941, als Warburg durch das Kultusministe- 
rium aufgefordert wurde, seinen Posten als Institutsdirektor zur Verfügung zu stellen - er wurde also hinausgeworfen, was zu diesem Zeitpunkt einem Todesurteil schon sehr nahe kam. Dank seiner Freunde und Gönner wurde Warburg jedoch bereits einige Tage später offiziell wieder ins Amt eingesetzt. $^{3}$ Er selbst schrieb dies bei verschiedenen Gelegenheiten unterschiedlichen Personen zu: Der Chirurg Ferdinand Sauerbruch soll beteiligt gewesen sein, doch haben wohl auch Warburgs Freund und Bewunderer Walter Schoeller und dessen Umfeld entscheidend dazu beigetragen, dass er seine Stellung behielt. Zudem wurde das KWI für Zellphysiologie zum kriegswichtigen „Wehrinstitut" erklärt. Auf welcher Grundlage diese Einstufung erfolgte, ist unklar, denn allen bekannten Quellen zufolge wurde in Warburgs Institut niemals für den Krieg gearbeitet, weder direkt noch indirekt (Werner 1991: 312). In jedem Fall war Warburgs Stellung für den Moment gerettet, doch konnte er nie sicher sein, wann der nächste, möglicherweise definitive Schlag gegen ihn erfolgen würde.

Wie Warburg diese Situation erlebte, wissen wir nur aus indirekten Quellen, etwa aus den Tagebuchnotizen seiner Schwester Lotte, die 1989 publiziert wurden (Rüskamp 1989). ${ }^{4}$ Schriftliche Dokumente von ihm selbst sind aus dieser Zeit bisher kaum bekannt. Um so überraschender ist daher der Fund ausführlicher und sehr persönlicher Tagebuchnotizen, in denen Warburg einen authentischen Bericht darüber gibt, wie er in seinem Ferienhaus auf Rügen die letzten Kriegswochen erlebte. Diese Aufzeichnungen sind in ihrer Art einmalig. Zwar führte Warburg über seine gesamte Forscherlaufbahn hinweg akribisch verschiedene Labor- und Notizbücher, in denen er experimentelle Daten notierte, relevante Beobachtungen zusammenstellte und Vorarbeiten zu Artikeln und Büchern festhielt. Doch nur in einem dieser Bücher finden sich private Bemerkungen von der Art, wie sie hier präsentiert werden. Auch über die Laborbücher hinaus sind keine vergleichbaren Aufzeichnungen von Warburg bekannt, weder aus den Jahren vor noch nach der Periode des Nationalsozialismus.

Warburgs Leben und Werk wurden schon mehrfach beschrieben, von sehr sachkundiger Hand und unter Einbezug zahlreicher Dokumente aus seinem Nachlass. Die in diesen Werken eingehend dargestellten und belegten Details seiner Biographie sollen daher nur insoweit wiederholt werden, als sie für den direkten Kontext und für ein Verständnis der Tagebuchnotizen relevant sind.

\section{Warburgs Situation in den Jahren 1944/45}

Warburgs Überlebensstrategie in den letzten Jahren des Nationalsozialismus bestand vorwiegend darin, einerseits die politischen Verhältnisse so weit wie möglich zu ignorieren, andererseits möglichst wenig Aufmerksamkeit auf 
sich zu ziehen. Er machte sich keine Illusionen über seine Stellung als „halbjüdischer" Wissenschaftler in einem nationalsozialistischen Staat, doch war er fest entschlossen, wie er bereits 1935 äußerte, sich aus Deutschland nicht „Von ein paar hergelaufenen Verbrechern“vertreiben zu lassen, sondern seine Arbeit so weit es eben ging unverändert fortzuführen (Rüskamp 1989: 252, Werner 1991: 285). Warburg war es sehr bewusst, dass seine Anwesenheit dem Regime diente und dass dies der Grund war für seine Sonderbehandlung. Wie er es seiner Schwester gegenüber im Jahr 1938 ausdrückte, hatte er „mit der Regierung eine Art Teufelspakt [geschlossen], einen sehr unanständigen, üblen“. Lotte Warburg beschreibt die Situation ihres Bruders folgendermaßen:

Sie halten ihn für die Auslandspropaganda und bezahlen ihm Devisen und alles, was er will, erhöhen auch seinen Etat, aber sie ignorieren ihn vollkommen im Inland. Da tun sie, als wäre er gar nicht da. Er wird nie zu einem Vortrag aufgefordert. Er sagt, er könne sich sehr gut denken, dass auf der Liste, die sie für alle Menschen haben, bei seinem Namen stehe: Noch zehn Jahre behalten für Auslandspropaganda. (Rüskamp 1989: 300)

Trotz dieser (und anderer) Einschränkungen wurde selbst nach Kriegsbeginn ohne Pause in Warburgs Institut an Fragen der Stoffwechselphysiologie gearbeitet, bis die wiederholte Bombardierung des Gebäudes dieser Tätigkeit ein Ende setzte. Im Sommer 1943 wurde das KWI für Zellphysiologie deswegen aus Berlin-Dahlem in die Uckermark verlegt, in das Schloss Seehaus in Liebenberg (Kreis Templin), das zu diesem Zweck umfassend renoviert wurde. ${ }^{5}$ Man mag vermuten, dass diese Ausgliederung des Instituts auch mit dem Ziel erfolgte, Warburg aus dem Blickfeld der Politik zu rücken, nachdem der Versuch, ihn abzusetzen, fürs erste erfolgreich abgewendet war (Henning 1987: 85). Warburg selbst hielt sich während der Renovierungsarbeiten vorwiegend in seinem Ferienhaus in Nonnevitz auf Rügen auf, wo er an dem Buch Schwermetalle als Wirkungsgruppe von Fermenten arbeitete, das 1946 erschien. Er schien an der weiteren Instandsetzung des Schlosses nur marginal interessiert zu sein. Angeblich erwartete (und erhoffte) er spätestens ab 1944 jederzeit die deutsche Kapitulation (Bücher 1983: 24).

$\mathrm{Zu}$ Warburgs Mitarbeitern in dieser Zeit gehörten Theodor Bücher, Walter und Marianne Christian, Fritz Kubowitz, Wilhelm und Luise Lüttgens sowie Erwin Negelein und die Brüder Richard und Alfred Triebener. Dazu kam in Liebenberg noch Kurt Christian, den Warburg in seinen Notizen erwähnt, dessen Funktion aber unklar ist. Möglicherweise war er ein Verwandter von Walter Christian. Fast alle waren schon seit langem für Warburg tätig: Insbesondere Walter Christian, Kubowitz und Negelein gehörten zu den ersten Mitarbeitern, die er überhaupt eingestellt hatte, damals noch als junger Abteilungsleiter am KWI für Biologie. Alle drei waren damit seit den 1920er Jahren ohne Unterbrechung bei Warburg. Auch Lüttgens arbeitete seit vielen Jahren in Warburgs Institut. Diese Kontinuität im Mitarbei- 
terstab mag zu dem angenehmen, vertrauten „Betriebsklima“ beigetragen haben, das Bücher in seinen Erinnerungen hervorhebt (Bücher 1983: 6f.). ${ }^{6}$

Um so härter traf es Warburg, als er in den Jahren 1943/44 persönlich denunziert wurde: Im Dezember 1943 erreichte ein Schreiben die Geheime Staatspolizei, in welchem Warburg unerlaubtes Autofahren, defätistische Bemerkungen, Deutschenhass und Anglomanie vorgeworfen wurden. Weiter wies der Schreiber darauf hin, dass Warburg in der "Wilhelmstrasse“ gedeckt werde. Die detaillierte Kenntnis von Warburgs Lebensweise, die aus dem Schreiben sprach, deutete darauf hin, dass dieser Brief aus dem Kreis seiner engsten Mitarbeiter stammte. Zudem war der Brief von Zehdenick aus abgeschickt worden, ein Nachbarort von Liebenberg. Im Februar 1944 erreichte ein weiteres, sehr belastendes Schreiben die Generalverwaltung der Kaiser-Wilhelm-Gesellschaft, unterzeichnet von Warburgs Mitarbeiter Fritz Kubowitz. ${ }^{7}$

Warburg konnte sich erneut mit Unterstützung verschiedener Freunde retten. Das Vertrauen in seine Mitarbeiter war jedoch zerstört - und, wie Bücher in seinem Nachruf bemerkt, auch die Atmosphäre innerhalb der Arbeitsgruppe (Bücher 1983: 10). ${ }^{8}$ Wie seinen Tagebuchnotizen aus dem Jahr $1945 \mathrm{zu}$ entnehmen ist, war Warburg nicht mehr bereit, sich in irgendeiner Weise für diejenigen Mitarbeiter einzusetzen, die er als Urheber der Affäre vermutete - nicht einmal, um sie vor dem nahezu sicheren Tod im Volkssturm zu bewahren: das waren neben Kubowitz auch Negelein sowie Walter Christian und seine Frau (wobei nicht belegt ist, dass Negelein und das Ehepaar Christian tatsächlich an der Denunziation beteiligt waren). ${ }^{9} \mathrm{Da}$ Warburg seine Gestapo-Akte persönlich vernichtet haben soll, als sie ihm von der sowjetischen Besatzung übergeben wurde, ist eine definitive Aufklärung dieser Affäre heute nicht mehr möglich (zum Beispiel Henning 1987: 102). Doch bilden diese Ereignisse den Hintergrund für den bitteren Ton von Warburgs Aufzeichnungen.

Der später berühmte Biochemiker Theodor Bücher (1914-1997) war zu dieser Zeit der einzige akademisch ausgebildete Mitarbeiter Warburgs. Er kam 1938 - zu einer Zeit, in der dies fast niemand mehr wagte - in Warburgs Labor, um hier seine Dissertation anzufertigen, und blieb auch danach im Institut tätig. Bücher wurde schon bald nach Ausbruch des Zweiten Weltkriegs zum Kriegsdienst eingezogen, doch hielt er den Kontakt zu Warburg aufrecht und soll ihn mit Kaffee und Zigaretten versorgt haben. Bücher kam aus höchst einflussreichem Hause: Sein Vater, Geheimrat Hermann Bücher (1882-1951), war zu dieser Zeit nicht nur Vorstandsvorsitzender der AEG und Mitglied im Aufsichtsrat zahlreicher Unternehmen, zum Beispiel der Krupp AG, sondern seit Mai 1942 zudem Mitglied des Deutschen Rüstungsrates. Warburg suchte dann auch Hermann Büchers Hilfe, um einige seiner Mitarbeiter für Forschungszwecke vom Kriegsdienst freizustellen. Ein ent- 
sprechendes Gesuch für Büchers Sohn Theodor wurde zwar abgelehnt, doch konnte immerhin ein verlängerter Urlaub erwirkt werden (Werner 1991: 315). Nach Theodor Büchers eigener Einschätzung hat ihm die Protektion durch Warburg das Leben gerettet (Bücher 1983: 27, Höxtermann/Sucker 1989: 144). Warburg gelang es weiterhin, seinen langjährigen Gefährten Jacob Heiss vor Kriegsdienst und Volkssturm zu bewahren. Ob Hermann Bücher daran beteiligt war, ist unklar. ${ }^{10}$

\section{Die Aufzeichnungen}

Die Tagebuchnotizen von Warburg finden sich in einem seiner zahlreichen Labor- und Notizbücher, die für die Zeit nach 1925 nahezu vollständig erhalten sind. ${ }^{11}$ Warburg begann das fragliche Notizbuch im Dezember 1940 in seinem Ferienhaus in Nonnevitz auf Rügen. ${ }^{12}$ Die ersten circa 150 Seiten enthalten Vorarbeiten zu seiner Monographie Schwermetalle als Wirkungsgruppe von Fermenten, die nächsten hundert Seiten sind leer. Erst ab Seite 277 finden sich die Aufzeichnungen, um die es hier geht: unter der Überschrift „Tagebuch. Ab 24. Februar 1945“. Zu diesem Zeitpunkt wohnten nur noch Lüttgens und seine Frau dauerhaft im ausgelagerten Institut in Liebenberg, aber auch Bücher hielt sich zumindest teilweise dort auf.

Aus welchem Grund und für wen Warburg diese Notizen verfasste ist unklar. Sie scheinen nicht zur Publikation bestimmt, haben keinen persönlichen Adressaten und, soweit bekannt, hat sich weder Warburg selbst noch irgend jemand sonst jemals auf diese Einträge bezogen. Vermutlich waren sie sogar Jacob Heiss unbekannt, der viele andere persönliche Dokumente nach Warburgs Tod vernichtete. ${ }^{13}$ Im Großen und Ganzen bestätigen die Notizen unser bisheriges Bild von Warburg, jedoch ergeben sich durchaus neue Facetten: So hatte er etwa keinerlei Bedenken, aufgrund einer persönlichen Kränkung langjährige Mitarbeiter in den Volkssturm zu schicken. Neu sind weiterhin die Details zu Theodor Bücher und seinem Vater, mit denen Warburg auch in diesen Monaten in engem Kontakt stand. Büchers Versuch, einen Teil der Laborinstrumente zu retten, war ebenfalls bisher nicht bekannt. Diese sachlichen Informationen sind in den Notizen klar beschrieben und es gibt keinen Grund, an ihrer Korrektheit zu zweifeln. Bei jeder Bewertung der Akteure und ihrer Handlungen ist dagegen stets zu bedenken, dass Warburg häufig in seiner Darstellung übertrieb und nicht selten die Fakten zu seinen Gunsten verbog.

Warburgs Aufzeichnungen werden im Folgenden vollständig transkribiert wiedergegeben. Die angegebenen Seitenzahlen entsprechen der Paginierung des Notizbuches. 


\section{/S. 277/ Tagebuch. Ab 24. Februar 1945.}

\section{/S. 278/ Nonnevitz, den 24. Februar 1945.}

Das Dahlemer Institut soll nun nicht Feldlazarett werden, sondern städt. Krankenhaus, und zwar eine Abteilung des Berliner Martin-Luther-Krankenhauses. Ober-Inspektor der Stadt sicherte zu, das Haus sollte möglichst geschont werden.

Mein Privathaus in Dahlem ist wieder von einer Kommission besichtigt worden, die Flüchtlinge aus d. Osten unterbringen will. Frau Schmidt telefonierte, sie habe die Gefahr „abgebogen“. Geheimrath Bücher bat um die Erlaubnis, in meinem Haus in Dahlem für sich u. seine Frau Lebensmittel zu deponieren. Er meint, im Fall eines russischen Vormarschs nicht auf seinem Gut (bei Friesack) bleiben zu können.

„Aussenstellen-Leiter“ meines Instituts ist jetzt Professor Nipper, Berlin-Dahlem. Er will ein Verzeichnis der Angestellten, von denen 30\% in Volkssturm I u. 70\% in Volkssturm II sollen. Geantwortet, dass die Einteilung von dem Kreisleiter Templin bereits vorgenommen worden ist. Wir haben $2=25 \%$ in I u. 6 in II. Kubowitz und Negelein sind in I. Kubowitz ist bereits im Einsatz in Frankfurt/Oder u. schrieb an mich, ob ich ihn nicht zurückholen könne. Man kann aber nicht heute einen Chef denunzieren u. ihn morgen bitten, ihm sein /S. 279/ Leben zu retten. Ich habe nichts unternommen.

Die Russen stehen in Pommern bei Stargard vor Stettin, in Brandenburg bei Küstrin u. Frankfurt vor Berlin. Was wird werden und wann? In Nonnevitz ist alles ruhig.

Nonnevitz, den 6. März 1945

Frau Lüttgens telefonierte, dass heute die Angestellten in Liebenberg vom Volks-Sturm eingekleidet wurden $u$. Waffen erhalten. Geheimrath Bücher sei gestern dagewesen $u$. habe mit seinem Sohn gesprochen. Doch wisse sie nicht was [er], u. was der Sohn vorhabe. Sie scheint zu glauben, dass Bücher doch noch etwas zu seiner "Sicherstellung" unternimmt. - Hier ist noch alles ruhig, obwohl die Russen schon am Ostufer des Stettiner Haffs sind. - Aufforderung in der Rügen'schen Leitung, die Fahrräder zu melden, die alle beschlagnahmt worden sind. - Lebensmittelrationen gekürzt, besonders Brot und Fett.

\section{/S. 280/ Nonnevitz, den 7. März 1945}

Gestern abend um 11 Uhr Sassewitz von Engländern schwer bombardiert. Bahnhöfe, Hafen u. Kaserne sollen zerstört sein, auch Schiffe mit Flüchtlingen, die im Hafen lagen. - Die Russen sollen schon in Usedom u. Wollin sein, ferner in Altdamm, Vorstadt von Stettin. - Ich habe gestern abend Bücher erlaubt, Apparate in das Nebengebäude des Instituts in Liebenberg nach Dahlem zu bringen. - Mit Frau Lüttgens telefoniert, die sich sehr gut bewährt. Lüttgens u. Frau sind die ersten, die ich wieder anstelle - falls ich etwas anzustellen habe!

Von Usedom nach Göhren auf Rügen sind 20 km. Ob die Russen von dort mit Fallschirmen nach Rügen können?

\section{/S. 281/ Nonnevitz, den 13. März 1945}

Liste an die Kreisleitung Templin eingereicht, in welcher Reihenfolge die Angestellten des Instituts zum aktiven Volkssturm abgegeben werden sollen:

Kurt Christian $\rightarrow$ Richard Triebener $\rightarrow$ Walter Christian $\rightarrow$ Alfred Triebener $\rightarrow$ Bücher $\rightarrow$ Lüttgens. 
Swinemünde vorgestern schwer bombardiert. - Küstrin soll von d. Russen genommen sein.

Nonnevitz, den 17. März 1945

Bücher telefonierte, dass die Trennung von Volkssturm I u. II aufgehoben ist; oder dass alle Angestellten jetzt in I sind. Liebenberger Zug, Müller Zugführer, Bücher Führer einer Gruppe. Frau Negelein war bei Bücher - „völlig ausgezehrt“ - ob wir nicht einen ErsatzMann für ihren Mann stellen können, der in Templin beim Volkssturm eingezogen ist; könnte auch irgend ein Mann von Falkenthal sein; brauchte nicht einmal Falkenthal sein, sondern könnte auch ein „Flüchtling“ sein. - Ich habe jede Intervention abgelehnt. Denn warum soll ich versuchen andere Menschen zum Schutz ihres Mannes vorzuschicken?

\section{/S. 282/ Nonnevitz, den 20. März 1945}

Bücher telefonierte von Liebenberg: Dr. Riedel von Osram war in Liebenberg. Es gäbe einen neuen Führerbefehl über das Verhalten bei Lähmung der Betriebe [?], infolge Herannahens des Feindes. Inhalt etwa

1) es soll im Betrieb nichts zerstört werden.

2) es soll eine Teil-Verlegung in Aussicht genommen werden, um die wichtigsten Akten und Apparate zu entfernen.

3) es soll ein Betriebs-Volkssturm gebildet werden, der die Betriebsmittel bis auf den letzten Mann verteidigt.

Können wir diesen Führerbefehl bekommen?

Bei [Ernst] Telschow angerufen. War nicht da. Büro K.W.G. angerufen. - Nein, gilt nur für Betriebe, die „Endfertigung“ liefern u. die ihre eigenen Volkssturmbataillone aufgestellt haben. Auskunft von Dr. Graue, der zufällig im Büro der K.W.G. war.

\section{/S. 283/ Nonnevitz, den 21. März 1945}

Bücher telefonierte aus Liebenberg, er könne eine Schweizer Holzbaracke bekommen. Habe die Absicht, seine Familie darin unterzubringen. Ich sagte, ich würde überlegen. Geht aber aus vielen Gründen nicht. Am nächsten Tag abgelehnt.

\section{Nonnevitz, den 28. März 1946}

Bücher zum Rüstungsamt nach Berlin geschickt mit der Bitte um Teilverlegungs-Auftrag (Ministerialrat Speer). Telefonierte, dass er den Auftrag erhalten hat für die Gegend Magdeburg-Stendal. Vater will Jagdhaus dort zur Verfügung stellen von A.E.G.-Director. Damit ist Bücher dann versorgt. Bleibt nur noch Lüttgens. Vielleicht kann man vom Rü-Amt Auftrag bekommen, dass Lüttgens beim Rest-Institut in Liebenberg bleiben muss. - In Rügen bisher alles ruhig. Nur immer mehr u. mehr Flüchtlinge aus Pommern am linken [Ufer] der Oder. [sic]

\section{/S. 284/ Nonnevitz, den 18. April 1945}

Am Donnerstag, d. 12. April ist Bücher mit Lastauto von Liebenberg losgezogen, ohne sich bei mir abzumelden. Alles war unkorrect 
1) er hat von dem Verlagerungsbefehl des Rüstungsamtes keine Abschrift gezeigt oder im Büro gelassen.

2) er hat kein Verzeichnis der von ihm fortgeführten Gegenstände hinterlassen

3) er hat ohne zu fragen, 10 Liter Alkohol eingepackt, wovon er 5 Liter nicht herausgegeben hat.

Ich nehme an, dass er nicht in die Letzlinger Heide gekommen ist. Doch hat die Verantwortung das Rüstungsamt.

Frau Lüttgens rief vorgestern an, dass die Russen wahrscheinlich kämen und verabschiedete sich von mir mit Dank. Gestern und heute aber haben wir wieder zusammen telefoniert $\mathrm{u}$. die Lage ist ruhiger.

[kein weiterer Eintrag]

\section{Nach April 1945}

Über die Situation in Liebenberg ab dem 15. April 1945 (bis September 1945) sind detaillierte Tagebuchnotizen von Lüttgens erhalten. Diese schließen somit direkt an die Aufzeichnungen von Warburg selbst an. Sie wurden bereits im Wortlaut in der dokumentarischen Biographie Warburgs von Petra Werner publiziert und sollen daher nicht wiederholt werden (Werner 1991: 326334, Dok. 124). Eine knappe Zusammenfassung mag genügen.

Insgesamt wurde die Situation zunehmend bedrohlicher: „Jeden Tag und jede Nacht oft drei Mal Fliegeralarm“, notierte Lüttgens am 15. April (Werner 1991: 326). Am 27. April telefonierte Lüttgens ein letztes Mal mit Warburg auf Rügen, am nächsten Tag begannen die Kämpfe direkt um Liebenberg und am 30. April 1945 übernahm die Rote Armee den Ort. Im Rahmen der Besetzung wurde Warburgs Institut im Schloss Seehaus nahezu vollständig ausgeräumt. Obwohl der russische Kommandant der besetzten Zone, Marschall Shukow, sich später persönlich für diese Plünderung entschuldigte und die Rückgabe der Gerätschaften anordnete, wurden Instrumente und Einrichtung nie wieder aufgefunden. Die KWG überließ daraufhin das Seehaus dem Zehdenicker Krankenhaus zur Nutzung. Der in Warburgs Tagebuch geschilderte Versuch von Theodor Bücher, zumindest einige Gerätschaften aus Liebenberg zu retten, schlug ebenfalls fehl, wie aus einem Brief von Bücher an Warburg vom Dezember 1945 hervorgeht. ${ }^{15}$ Auch diese Instrumente waren also verloren. Im Juni 1945 wurde schließlich auch das Institutsgebäude in Berlin-Dahlem vom Berliner Oberkommando der Amerikaner beschlagnahmt. Daraufhin ließ Warburg alle Angestellten des Instituts entlassen (Werner 1991: 338).

Warburg selbst blieb bis September 1945 auf Rügen, wie er seiner Schwester Lotte im Januar 1946 schilderte:

Bis Ende September war ich mit Jacob [Heiss], den ich mit viel Kunst vom Militärdienst und vom Volkssturm gerettet habe, und dem es gut geht, in Rügen. Wir sind dann mit russischen Autos nach Dahlem gefahren worden, mit allen unseren Sachen. Die beiden Pferde hat mir Marshall Shukow vor vier Wochen aus Rügen geholt, der mich fragte, ob ich einen 
Wunsch hätte, nachdem ich in Babelsberg mit ihm gegessen hatte. Nun sind die Pferde wieder in Düppel und alles ist in meinem Privatleben wie früher. Weniger günstig sieht es zur Zeit mit dem wissenschaftlichen Arbeiten aus. ${ }^{16}$

Den Personen, die Warburg der Denunziation verdächtigte - zumindest Kubowitz und dem Ehepaar Christian -, verzieh er nie. ${ }^{17} 1946$ wandte sich Christian an Warburg mit der Bitte um ein Arbeitszeugnis für sich und seine Frau; dieser Brief blieb unbeantwortet. Als Marianne Christian nach dem Tod ihres Mannes im Jahr 1955 diese Bitte für sich wiederholte, schickte Warburg lediglich eine formelle Arbeitsbescheinigung. ${ }^{18}$ Hingegen hatte Warburg selbst sich mit einem eingeschriebenen Brief an Karl Lohmann gewandt, bei dem Christian im Jahr 1946 eine Anstellung gefunden hatte. Darin schrieb Warburg an Lohmann Folgendes:

Sehr geehrter Herr Lohmann

In den Bestimmungen der Alliierten Kommandantur, die heute in den Tageszeitungen veröffentlicht worden sind und die der Säuberung des öffentlichen Lebens dienen, heisst es:

2. Personen sind aus ihren Stellungen zu entlassen, wenn sie:

I. [von Warburg frei gelassen]

II. [von Warburg frei gelassen]

III. Naziverbrechen, Rassenverfolgungen oder unterschiedliche Behandlung angestiftet oder an ihnen bejahend teilgenommen haben.

Dieser Abschnitt III trifft auf das Ehepaar Christian in so hervorragendem Masse zu, dass er eigens für sie beide aufgenommen worden zu sein scheint. Dabei betrifft die Verfolgung mittels Denunziation nicht nur meine Person, sondern auch 2 Holländer, die in Liebenberg bei mir angestellt waren. Die Denunziationen liegen zum Teil in Photokopie vor. 3 Zeugen, die das Ehepaar Christian beobachtet haben, sind zur eidlichen Aussage bereit und zur Verfügung. - Ich bin der Meinung, dass beide mir zu grossem Dank verpflichtet sein müssen, wenn sie nicht als kriminell in ein Lager der russischen oder amerikanischen Militärregierung gebracht werden. Es befindet sich ferner eine holländische Militärmission in Berlin, die für diese Fälle grosses Interesse hat.

Mit vielen Grüssen bin ich Ihr Otto Warburg. ${ }^{19}$

Wie Christian hatte auch Kubowitz erfolglos um ein Arbeitszeugnis gebeten, wie aus einem Brief an Warburg aus dem Jahr 1955 hervorgeht:

Anlässlich einer persönlichen Vorsprache bei Ihnen im Jahre 1946 verweigerten Sie mir, vertreten durch Herrn Heiss, sowohl die Schlusseintragung im Arbeitsbuch, als auch die Ausstellung einer Tätigkeitsbescheinigung. Ich bitte Sie hiermit höflichst, mir diese Bescheinigung auszustellen und sie mir freundlicherweise zuschicken zu lassen. ${ }^{20}$

Kubowitz wies Warburg weiter darauf hin, dass dieser dem Gesetze nach dazu verpflichtet sei, eine solche Bestätigung „über die Dauer der Tätigkeit bei Ihnen und über de Art der Beschäftigung und des Dienstverhältnisses, auf Anforderung auszustellen“. ${ }^{21}$ Falls Warburg die Ausstellung der Bescheinigung verweigere, werde er Klage beim Arbeitsgericht erheben. Warburgs Antwort darauf ist leider nicht erhalten. 
Mit Theodor und Hermann Bücher hingegen blieb Warburg auch nach 1945 in Kontakt. Unter anderem bemühte er sich, bei seinem Aufenthalt in den Vereinigten Staaten in den Jahren 1948/49 eine Stelle für Theodor Bücher zu finden. ${ }^{22}$ Für Hermann Bücher stellte Warburg im Januar 1946 ein entlastendes Zeugnis über dessen Haltung während der NS-Periode aus, in welchem er unter anderem Büchers Einsatz zur Rettung von Warburgs Stelle im Jahr 1941 hervorhob. ${ }^{23}$

Theodor Bücher war vermutlich derjenige, der über die Ereignisse um die Denunzierung und ihre Folgen am besten informiert war, doch hielt er sich in der Öffentlichkeit diesbezüglich sehr bedeckt. 1947 kam es zu einem Briefwechsel mit Krebs, in dem es vor allem um Warburgs polemisch geschriebene Monographie Schwermetalle als Wirkungsgruppe von Fermenten ging - eben diejenige Monographie, an der Warburg in den letzten Kriegsjahren in Nonnevitz arbeitete. Bücher erklärte Warburgs Tonfall in diesem Buch mit der schwierigen, persönlichen Situation, in der dieser sich befand:

\begin{abstract}
Warburg's book was written in days of extraordinary agitation in great as well as in small events: at the beginning of the final phase of the war, of the emptying and evacuation of the institute from Dahlem to Liebenberg (summer 1943-spring 1944), a time, which was filled with dreadful, inevitably escalating disputes internally between Warburg and his coworkers, quarrels which came to a horrifying end when Kubowitz and Negelein were called up to join an active Volkssturm squadron and moved to the collapsing front at the Oder. (Bücher 1983: 27)
\end{abstract}

Während Bücher also von „furchtbaren, zwangsläufig eskalierenden Streitereien“ zwischen Warburg und seinen Mitarbeitern spricht, ist von einer Denunziation keine Rede. Bücher erwähnt weiterhin in seinem Brief an Krebs, dass er in dieser Zeit in engem Kontakt stand mit Warburg und sich erfolglos bemühte, Warburg aufzumuntern. „This year's visit was under the same fatal star of condemnation of his oldest collaborators", beendet Bücher diese Passage seines Briefes recht dunkel und mehrdeutig. ${ }^{24}$ Dass Krebs von der Reichweite der "Streitereien“ nichts wusste, belegt auch ein Brief von ihm an Warburg aus demselben Jahr 1947, in dem er sich nach den langjährigen Mitarbeitern Warburgs erkundigte:

I was very glad to hear from Bücher that you are in good health and living in relative comfort. He mentioned that he had seen you recently and that you are engaged in writing two monographs. These will be eagerly awaited by many people over here. Bücher never mentioned what has become of Negelein, Kubowitz and Christian. I hope they are well.

Warburgs Antwort ist leider nicht erhalten, doch ist nicht anzunehmen, dass er ausführlich zu diesem Punkt Stellung bezog. 


\section{Anmerkungen}

1 Aus der Fülle von biographischen Arbeiten zu Otto Warburg siehe zum Beispiel (in chronologischer Reihenfolge): Krebs 1972, 1979, Bücher 1983, Henning 1987, Werner 1988, Höxtermann/Sucker 1989, Werner 1991, Höxtermann 2001.

2 Zum Schicksal anderer Wissenschaftler, nur allein in Warburgs Disziplin, siehe etwa Deichmann 2001. Die Geschichte der Kaiser-Wilhelm-Gesellschaft im Nationalsozialismus war Gegenstand eines breit angelegten Forschungsprojektes. Für die reichen Erträge siehe insbesondere die von Reinhard Rürup und Wolfgang Schieder im Auftrag der Präsidentenkommission herausgegebenen Buchreihe gleichen Namens im Wallstein Verlag.

3 Die Episode wurde im Detail recherchiert und findet sich zum Beispiel dargestellt in Werner 1988: 262-263, 1991: 305-312, Henning 1987: 99f. sowie in Eckart Hennings Einleitung des Findbuchs zu Warburgs Nachlass im Archiv der Max-Planck-Gesellschaft (= MPG-Archiv; III. Abt., Rep. 1), siehe Henning 1985. Siehe für eine Zusammenfassung auch Deichmann 2001: 111.

4 Lotte Warburgs Aufzeichnungen sind jedoch nur sehr bedingt zuverlässig, zudem sind die publizierten Notizen nur Auszüge aus dem Gesamtbestand der Tagebücher.

5 Das Folgende stützt sich insbesondere auf die Darstellungen in Henning 1987, Werner 1991 sowie Höxtermann/Sucker 1989. Eine wertvolle Quelle für diese Zeit in Warburgs Leben sind auch die Erinnerungen von Theodor Bücher (1983), Warburgs einzigem Doktoranden in dieser Zeit.

6 Bücher gibt weiterhin Detailinformationen insbesondere zu Kubowitz, Negelein und Christian. Er betont dabei, dass alle drei zu Unrecht häufig abwertend als „technische Mitarbeiter" Warburgs bezeichnet werden, obschon die Qualität ihrer (eigenverantwortlichen) Arbeit dem Vergleich mit vielen anerkannten Biochemikern der Zeit durchaus standhält.

7 Eine eingehende Beschreibung der Ereignisfolge findet sich zum Beispiel in der Einleitung zu Henning 1985. Der Brief von Kubowitz ist im Archiv der Max-Planck-Gesellschaft erhalten. Vergleiche zu der Episode auch Werner 1988: 269-271, 1991: 316, Höxtermann/Sucker 1989: 143.

8 Theodor Bücher bestätigt hier, dass Kubowitz als Spitzel tätig war: „Not without some fault on his own side, this outstanding man of firm character [Kubowitz, K.N.] got caught in the net of Gestapo inquiries about Otto Warburg. This extremely dangerous incident happened in the course of the removal of the Institute to Fürst Eulenburg's Schloss Seehaus in Liebenberg. It was finally suppressed by very great efforts, but nevertheless weighted heavily on the working climate in the group."

9 Diese Personen bezichtigte Warburg am 5. August 1946 als NS-Denunzianten und verfolgte sie dementsprechend. Siehe die Angaben in Henning 1985: 17.

10 Jacob Heiss (1899-1984) kam 1919 als „Bursche“ zu Warburg und führte ihm seither den Haushalt und lebte mit Warburg als Freund, Gefährte, Sekretär - mit hoher Wahrscheinlichkeit auch als Partner (Werner 1988: 162-163, Krebs 1979: 110).

11 Warburgs Labor- und Notizbücher sind größtenteils einsehbar im Archiv der Berlin-Brandenburgischen Akademie der Wissenschaften (BBAW-Archiv). Dort befindet sich auch das fragliche Notizbuch, aus dem hier zitiert wird. Weitere Laborbücher sind im Archiv der Max-Planck-Gesellschaft (MPG-Archiv Berlin) erhalten. Ich danke den Mitarbeitern und Mitarbeiterinnen beider Archive für die tatkräftige Unterstützung meiner Recherchen sowie für die Erlaubnis zur Publikation dieser Quelle.

12 BBAW-Archiv, NL Warburg 2, Titel des Notizbuches: „Nonnevitz, ab 23.12.1940“.

13 Persönliche Mitteilung von Marion Kazemi, MPG-Archiv, Berlin.

14 Während die zum „Volkssturms II“ eingeteilten Personen (aus „kriegswichtigen Berufen“) nur kurzzeitig und in Heimatnähe eingesetzt werden sollten, konnte der „Volkssturm I“ bis zu sechs Wochen am Stück und auch in entfernten Gebieten eingesetzt werden. 
15 Bücher an Warburg, 26. Dezember 1945. Der Brief ist im Wortlaut abgedruckt in Werner 1991: 335-337, Dok. 126.

16 Warburg an Lotte Warburg, 13. Januar 1946. Ebd.: 355f., Dok. 128.

171973 schrieb Hans Krebs an Negelein, der Warburgs Denunziations-Verdacht anscheinend nie verwunden hatte: „Lieber Herr Negelein! Haben Sie vielen Dank für Ihren Brief. Ich habe volles Verständnis für Ihre Einstellung gegenüber Warburg, war ich doch selbst zeitweise ein Opfer seiner unbegründeten Verdächtigungen, was mir sehr schmerzlich war. [...] In meinem Nachruf habe ich auch erwähnt (S. 672), dass viele seiner Bewunderer unter seinen paranoidischen Vorstellungen gelitten haben." (Werner 1991: 319)

18 Siehe für beide Schreiben BBAW-Archiv, NL Warburg, 191.

19 Ebd., NL Warburg, 1113.

20 Ebd., NL Warburg, 550.

21 Ebd.

22 Siehe zum Beispiel den Brief von Hermann Bücher an Warburg, 18. Dezember 1948 (MPGArchiv; III, 1, 157), sowie den Brief von Theodor Bücher an Warburg, 6. August 1948 (MPG-Archiv; III, 1, 158).

23 Siehe das Zeugnis von Otto Warburg für Hermann Bücher, 20. April 1946 (BBAW-Archiv, NL Warburg, 1076). Aus dem Dokument wird zitiert in Werner 1991: 303. Siehe für dieses Zeugnis auch den Brief von Warburg an Hermann Bücher, 1946 (kein weiteres Datum: BBAW-Archiv, NL Warburg, 1054). Warburg stellte indessen auch Victor Brack als Dankesdienst für dasselbe „Geschäft“ (das heißt für die Rettung seiner Stellung als Direktor im Jahr 1941) ein Unbedenklichkeitszeugnis aus, obschon dieser als Mitarbeiter der Reichskanzlei nachweislich am Aufbau von Todeslagern, an mobilen Vergasungswagen, an Zwangssterilisationen und dem berüchtigten „Euthanasie“-Programm T 4 beteiligt war (siehe u.a. Werner 1991: 312). Brack wurde dennoch in den Nürnberger Ärzteprozessen zum Tode verurteilt; das Urteil wurde 1948 vollstreckt.

24 „I know him [Warburg] from many evening talks in Liebenberg in which I, living under the same roof with Warburg, tried with completely insufficient means to calm him down and to fight his bad spirits." (Bücher 1983: 27)

25 Hans Krebs an Warburg am 12. Mai 1947, BBAW-Archiv, NL Warburg, 542.

\section{Literatur}

Bücher, Theodor, 1983. Otto Warburg. A Personal Recollection. In: Horst Sund und Volker Ullrich, Hg., Biological Oxidations. Berlin u.a.: Springer, 1-29.

Deichmann, Ute, 2001. Flüchten, Mitmachen, Vergessen. Chemiker und Biochemiker in der NS Zeit. Weinheim: Wiley-VCH .

Henning, Eckart, 1985. Findbuch zu Warburgs Nachlass im Archiv der Max-Planck-Gesellschaft (MPG-Archiv; III. Abt., Rep. 1) [nicht publiziert, einzusehen im Archiv].

Henning, Eckart, 1987. Otto Heinrich Warburg. Der „Kaiser von Dahlem“. In: Wilhelm Treue und Gerhard Hildebrandt, Hg., Berlinische Lebensbilder. Bd. 1: Naturwissenschaftler. Berlin: Colloquium Verlag, 299-316.

Höxtermann, Ekkehard/Sucker, Ulrich, 1989. Otto Warburg. Leipzig: Teubner.

Höxtermann, Ekkehard, 2001. Otto Heinrich Warburg (1882-1970). In: Ilse Jahn und Michael Schmitt, Hg., Darwin \& Co. Eine Geschichte der Biologie in Portraits. Bd. 2. München: Beck, 251-274.

Krebs, Hans, 1972. Otto Heinrich Warburg 1883-1970. Biographical Memoirs of Fellows of the Royal Society, 18, 629-699.

Krebs, Hans, 1979. Otto Warburg. Zellphysiologe, Biochemiker, Mediziner 1883-1970. Stuttgart: Wissenschaftliche Verlagsgesellschaft.

Rüskamp, Wolf, 1989. Eine vollkommene Närrin durch meine eigenen Gefühle. Aus den Tagebüchern der Lotte Warburg 1925 bis 1947. Bayreuth: Druckhaus Bayreuth. 
Werner, Petra, 1988. Otto Warburg. Von der Zellphysiologie zur Krebsforschung. Berlin: Verlag Neues Leben.

Werner, Petra, 1991. Ein Genie irrt seltener. Otto Heinrich Warburg, ein Lebensbild in Dokumenten. Berlin: Akademie Verlag.

\section{Kärin Nickelsen}

Abteilung Wissenschaftstheorie und Wissenschaftsgeschichte Institut für Philosophie

Universität Bern

Sidlerstr. 5

CH-3012 Bern

E-Mail: kaerin.nickelsen@philo.unibe.ch 